\title{
„Pädiatrie komplex“
}

\section{Jahrestagung der Österreichischen Gesellschaft für Kinder- und Jugendheilkunde}

Die 59. Jahrestagung der ÖGKJ in Salzburg fand vom 29.09. bis 02.10.2021 im Salzburg Congress in Präsenz statt. 640 interessierte Teilnehmer aus Österreich und den angrenzenden Nachbarländern fanden sich bei der Tagung ein.

Das Hauptthema „Pädiatrie komplex" deutet an, wie wichtig im Fach Kinder-und Jugendheilkunde die Vernetzungen sowohl intern, als auch extern für die bestmögliche Qualität der Patientinnen und Patienten sind. Die Komplexität drückt aus, dass vielteilige inter- und multiprofessionelle und biopsychosoziale Aspekte auch für eine „personalisierte Präzisionsmedizin" unabdingbar sind. Das Thema wurde in der Spitzenmedizin, aber auch in der Ordination und an den Schnittstellen zu den Nachbardisziplinen der Pädiatrie sowie auch in kritischen Lebensphasen beleuchtet.

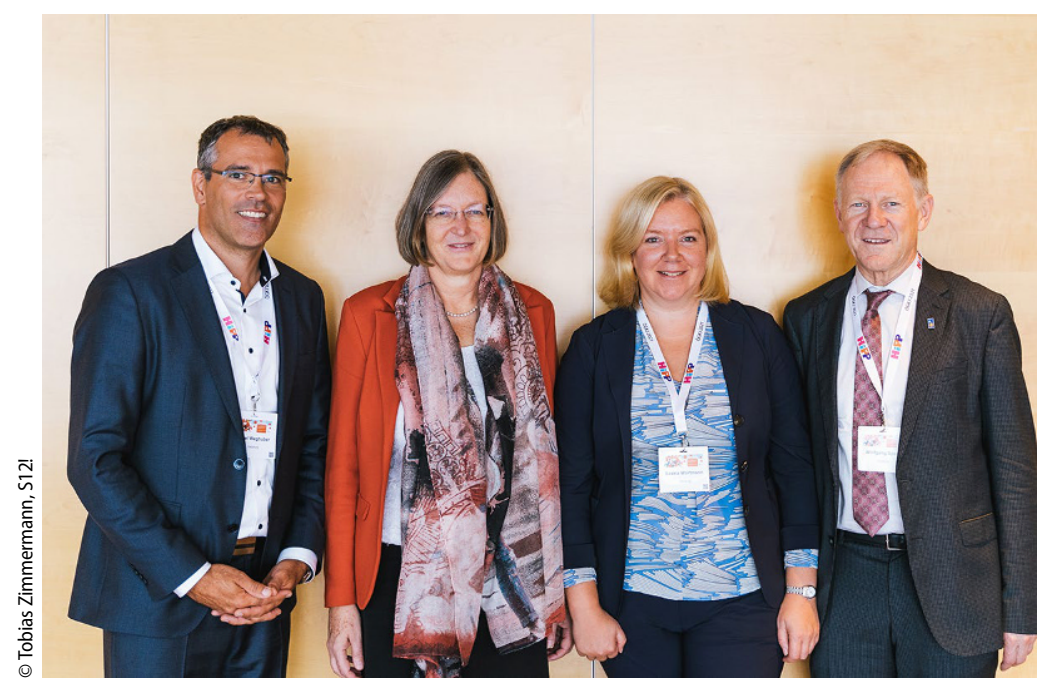

\ Pressegespräch zur ÖGKJ-Tagung 2021: v. I. n. r.: Daniel Weghuber, Daniela Karall, Saskia Wortmann, Wolfgang Sperl

\section{Unterschiedliche Lebensabschnitte} Das Fach Kinder- und Jugendheilkunde ist somit das ganze Gegenteil von einfach. Komplex bedeutet, dass in diesem Fach unterschiedliche Lebensabschnitte beinhaltet sind, unterschiedliche Organsysteme und dass im Gegensatz zur Erwachsenenmedizin eine sehr umfassende, Lebensabschnitt-betreuende Medizin betrieben wird. Zudem kommen die modernsten biochemischen Methoden im Bereich Forschung und auch der gesamten Molekulargenetik und der angeborenen Störungen zur Anwendung.

Die Schnittstellen der Pädiatrie mit Partnerfächern sind vielfältig wie Kinder-Chirurgie, Perinatologie, Augenheilkunde, HNO, Kinder-Anästhesie, Kinder-Orthopädie, Dermatologie, Radiologie u.a.m. Es wird regelmäßig mit den Erwachsenenmedizinern zusammengearbeitet.

(n)


kungen entdeckt, die Eingang in die medizinische Fachliteratur bekommen.

\section{Murmeltiersprechstunde}

Die Murmeltiersprechstunde unter der Leitung von Dozentin und „Forscherin des Jahres" OA Dr. Saskia Wortmann, Leiterin der Stoffwechselambulanz der Universitätsklinik für

Kinder- und Jugendheilkunde Salzburg ist eine multidisziplinäre Tagesklinik, wo seltene Entwicklungsstörungen zeit- und ressourcensparend diagnostiziert und mit personalisierte Präzisionsmedizin behandelt werden. "Dr. Murmel" ist das Maskottchen, das die Familien auf der Suche nach einer Diagnose begleitet.

Voraussetzung für einen Termin in der Murmeltiersprechstunde ist eine bisher ungeklärte Entwicklungsstörung. Das Spektrum der Entwicklungsstörungen betrifft rund ein Prozent aller Kinder, die Ursache ist überwiegend genetisch.
Die Zahlen und Erfolge aus der Murmeltiersprechstunde, die für immer mehr Patientinnen und Patienten lebensverändernd sind, belegen, dass die Uniklinik mit dieser Einrichtung auf einem goldrichtigen Weg ist: Bei bisher 43 teilnehmenden Kindern konnten in 28 Fällen der krankheitsverursachende genetische Fehler entdeckt und somit eine Diagnose gestellt werden. Bei sechs kleinen Patientinnen und Patienten (14\%) wurde eine maßgeschneiderte Therapie eingeleitet. Jeder einzelne Erfolg macht das behandelnde Team aus Kinder- und Jugendärztinnen und -ärzten, Humangenetikerinnen und -genetikern und Biochemikerinnen und -chemikern, stolz und spornt es an, in der personalisierten Präzisionsmedizin laufend neue Schritte zu gehen. Dieser unermüdliche Einsatz und die Erfolge geben auch den Familien enorm viel Kraft. Vom Erstgespräch bis zum Befund dauert es durchschnittlich sechs Monate, hiermit wird der früher oft jahrelange Weg zu einer Diagnose für die betroffenen Familien stark verkürzt.

\section{Telepräsenz-Roboter AVATAR „AV1“}

„Er ist gerade einmal 30 Zentimeter groß, wiegt nur zwei Kilogramm und kann für schwer erkrankte Patientinnen und Patienten Erstaunliches bewirken", berichtete Univ. Prof. Dr. Daniel Weghuber, Vorstand der Universitätsklinik für Kinder- und Jugendheilkunde Salzburg. Wenn ein Kind für längere Zeit ins Spital muss oder ein Reha-Aufenthalt notwendig ist, sitzt der Avatar auf dessen Platz in der Stammklasse. Das betroffene Kind kann sich via Tablet zuschalten und virtuell sogar "aufzeigen": Dann beginnt das Licht auf der Oberseite des Avatars zu blinken. Sogar seine Emotionen, sei es Freude, Traurigkeit oder Neugier, kann das Kind über die „Roboter-Augen" kommunizieren und so Teil der Klassengemeinschaft sein. Der ${ }_{\text {,AV1 }}$ “ bleibt nicht nur am Platz

Hier steht eine Anzeige. 
sitzen, er begleitet die anderen Kinder genauso in Pausen, beim Turnen oder bei Ausflügen.

Der Regelunterricht findet während der Erkrankung weiterhin in der Heilstättenschule des Klinikums statt, wo weitere elektronische Mittel schon lange vor der Pandemie zum Schulalltag gehörten. „Es geht beim Avatar vor allem darum, soziale Isolation zu verhindern", so Daniel Weghuber, neuer Vorstand der Salzburger Uniklinik für Kinder- und Jugendheilkunde. Kinder werden dank High-Tech-Unterstützung nicht mit voller Wucht aus ihrer Klassengemeinschaft gerissen. Infrage kommen für dieses Unterstützungstool vor allem Patientinnen und Patienten aus der Onkologie, der Psychosomatik, der REKIZ-Station, aber auch an der Universitätsklinik für Kinder- und Jugendpsychiatrie. Weghuber:,Personalisierte Präzisionsmedizin kümmert sich auch um das Beziehungsumfeld der Patientinnen und Patienten. Hier bedienen wir uns auch moderner Technik."

Das System, das ursprünglich aus Norwegen stammt, wurde in Österreich von der Wiener Heilstättenschule aufgegriffen und in einem vom Bundesministerium für Bildung, Wissenschaft und Forschung beauftragten Forschungsprojekt ausgerollt. Neben der MedUni Wien und der Alpen-AdriaUniversität beteiligt sich die Klinik für Kinder- und Jugendheilkunde der Paracelsus Medizinischen Privatuniversität. Weghuber: „Ein erster Probelauf ist im Sommer erfolgt, unsere Erfahrungen daraus sind sehr positiv. Wir wollen jetzt mit dem Projekt starten. "Vier Avatare sollen zur Verfügung stehen. Die Salzburger Landeskliniken, die 2021 zum „Jahr der Digitalisierung“ ausgerufen haben, finanzieren den Ankauf mit. Auch vom Ministerium wurde eine weitere Unterstützung bei der Ausrollung bereits signalisiert.

\section{COVID-Impfschutz für Kinder und Jugendliche}

Insbesondere in den letzten eineinhalb Jahren haben wir im Rahmen der Corona-Pandemie erlebt, wie Maßnahmen die Altersgruppe unter 18 Jahren besonders betroffen haben, obwohl sie selbst gar nicht die primäre Risikogruppe für die SARS-CoV-2-Infektion darstellt.,"Kinder und Jugendliche mussten die Folgen der Maßnahmen voll mittragen und hatten wohl die geringste Lobby", so Ao. Univ.-Prof. Dr. Daniela Karall, Präsidentin der Österreichischen Gesellschaft für Kinder- und Jugendheilkunde. „Auch in dieser Altersgruppe ist die COVID-Impfung nun eine anzubietende und sichere Option und Lösung."

Im Mai 2021 wurde der BioNTech/Pfizer mRNA Impfstoff für die Altersgruppe 12-15 Jahre zugelassen, im heurigen Herbst rechnen wir mit der Zulassung für die Altersgruppe 5-11 Jahre (Dosis auf 1/3 jener Dosis, die bei Jugendlichen über 12 Jahren verabreicht wird, angepasst).

Wie bei allen Impfungen wird auch bei der COVID-Impfung zwischen Impfreaktionen und Nebenwirkungen unterschieden. Bei Kindern und Jugendlichen werden ähnliche Impfreaktionen wie bei Erwachsenen beobachtet: Diese sind Schmerzen an der Einstichstelle, Kopf- und Gelenksschmerzen, Fieber und Schüttelfrost, welche ein bis drei Tage andauern können. Diese sind bei Bedarf gut mit z. B. Paracetamol oder Ibuprofen behandelbar.

Impfreaktionen sind ein Zeichen dafür, dass das Immunsystem sich mit dem Impfstoff auseinandersetzt, was ja gewünscht ist.

Im Spektrum der Nebenwirkungen wird im Kindes- und Jugendalter von Herzmuskelentzündungen berichtet. Wichtig ist, dass diese Entzündungen bei den Impflingen bisher unkompliziert verlaufen sind - ganz im Gegensatz zur Herzmuskelentzündung, welche aufgrund von Corona-Erkrankung entstehen kann. Der Schweregrad ist bei einer durchgemachten Infektion deutlich höher.
Im Rahmen einer sinnvollen RisikoNutzen-Abwägung ist es aus mehreren Gründen wichtig, dass Kinder und Jugendliche auch die Option zur Impfung bekommen - immer gemäß der Zulassung.

Karall weiter: „Aus allen Daten, die uns vorliegen, befürworten wir als ÖGKJ die Impfung für Kinder und Jugendliche derzeit ab zwölf Jahren, in Zukunft gemäß der Zulassung ab 5 Jahren mit angepasster Dosis. Betont werden sollte, dass es sich um eine frei-

Hinweis des Verlags. Der Verlag bleibt in Hinblick auf geografische Zuordnungen und Gebietsbezeichnungen in veröffentlichten Karten und Institutsadressen neutral.

Paediatr. Paedolog. 2021 · 56:310-312 https://doi.org/10.1007/s00608-02100950-1

(c) Springer-Verlag GmbH Austria, ein Teil von Springer Nature 2021

willige Entscheidung handelt, weil es in Österreich keine Impfpflicht gibt. Die Sorgen und Ängste, dass die Impfung unfruchtbar macht, das Erbgut verändert oder sogar tödlich ist, sind nicht haltbar."

Seitens der ÖGKJ wurde ein Elternbrief $^{1}$ formuliert, der in einfacher und verständlicher Form informieren soll, warum sich die ÖGKJ (auch) für die COVID-Impfung von Kindern und Jugendlichen ausspricht.

\section{Zur Person des Tagungs- präsidenten}

Die 59. Jahrestagung bildete zudem den Rahmen für den Abschied von Univ.-Prof. Dr. Wolfgang Sperl, der Ende Mai als Vorstand der Universitätsklinik für Kinder- und Jugendheilkunde emeritiert wurde und nun als Rektor der Paracelsus Medizinischen Privatuniversität sowie weiterhin auch als Vizepräsident der Österreichischen Gesellschaft für Kinder- und Jugendheilkunde agiert.

1 www.paediatrie.at/images/Covid19/eltern-
brief.pdf 